\title{
Full structure of the thermal plasma including the ion isothermal drag
}

\author{
M. Khoram ${ }^{1}$ (I).S. F. Masoudi²
}

Received: 26 July 2019 / Accepted: 23 December 2019 / Published online: 7 January 2020

(c) The Author(s) 2020

\begin{abstract}
In order to study the ion temperature effect on the space-charge structure and the plasma variables on the wall, the ion thermal force has been added to the ion motion equation in the plasma fluidal model. In the eigenvalue problem of plasma, the plasma equations are numerically solved in a whole area from the plasma center to the wall and it is displayed that the ion temperature has significant effects on the plasma structure and floating variables. However, the fluidal theory of plasmas introduces a singular point among the space charge of plasma boundary layer if the static pressure and the inertial mass of the thermal ions are taken into account at the same time. Finding a full numerical solution for the thermal plasma equations needs to cross the singular point. The singular point and how crossing the point will be depicted too.
\end{abstract}

Keywords Isothermal drag $\cdot$ Ion temperature $\cdot$ Full solution $\cdot$ Singularity point

\section{Introduction}

The subject of plasma boundary layer is nearly as old as the subject of plasma body and is very important in many fields of plasma physics and plasma technology including fusion researches, plasma diagnostics and material plasma processing [1-4]. For example, in plasma immersion ion implantation (PIII) that is an effective technique for semiconductor fabrication and material processing, knowing the structure of plasma boundary layer plays the main role [5-7].

The mathematical examination of plasmas near walls and electrodes has attracted considerable interest and has been turned to the main issue of many explorations [8-19]. Among the several parameters that their effects have been studied on the plasma variables and structure, the ion temperature effects in thermal plasmas are significant and have been examined by many authors [20-35].

It is apparent that the plasma fluid equations introduce singularities among the quasi-neutral plasma core if the

M. Khoram

m.khoramabadi@srbiau.ac.ir

S. F. Masoudi

masoudi@kntu.ac.ir

1 Department of Basic Sciences and Engineering, Borujerd Branch, Islamic Azad University, 6915136111 Borujerd, Iran

2 Department of Physics, K. N. Toosi University of Technology, P. O. Box 15875-4416, Tehran, Iran static pressure and the inertial mass of the thermal ions are taken into account at the same time [20,21,36]. Since the quasi-neutral one-fluid equations represent an acceptable smooth solution without any singularity, it follows that the singularity represented in the two-fluid equations of thermal plasmas should be obliterable [20, 36-38].

Friedman and Levi [36] have solved the two-fluid equations of thermal plasmas using a so-called neutral approach. Using a perturbation method, Franklin [20] has analyzed the same equations, and he got the ratio $\tau_{\mathrm{i}} / \tau_{\mathrm{e}}$ as the perturbation parameter with $\tau_{\mathrm{i}}$ and $\tau_{\mathrm{e}}$ as the ion and electron absolute temperature, respectively. Valentini [21] has tried to remove the irregularities at the singular point and its near proximity, and has represented a systematic method to prevail the singularity problems via numerical calculations.

In the present paper, using the boundary conditions at the plasma center and on the wall, the two-fluid equations are solved throughout the whole plasma for some values of the ion temperature in order to obtain a precise description of the plasma boundary layer structure. In order to find a smooth solution, using an approximation method, the main equations are analyzed in an velocity interval around the singular point. The effects of ion temperature and ion generation rate are investigated on the boundary layer structure and its floating variables.

In "Model and equations" section of this inquiry, warm plasmas incorporating the ion isothermal drag are formulized. The asymptotic limits of plasma including the 
plasma asymptotic and sheath asymptotic limits are introduced and are analytically solved in the plasma and sheath scales, respectively, in "Asymptotic and full solutions" section. Also the plasma full equations in the plasma scale, including the smallness parameter $\alpha$ and their numerical solutions will be presented in this section. The summary and results section summarizes paper derivations.

\section{Model and equations}

A thermal plasma confined between two metal plane walls located at $z= \pm L$ is considered to investigate the thermal behavior of plasma variables. Whereas the electrodes are flat and there are no sidewalls, a one-dimensional study is considered. Indeed, the plasma vessel is unlimited laterally and a longitudinal plasma is formed just between the electrodes, so the plasma is wasted at the both ends on the electrodes and there is not any lateral instability for plasma. The warm ions are modeled using the fluidal approximation of plasma. Since they are in thermal equilibrium, the electrons are described using the Boltzmann's relation. Also the plasma is symmetrical around the central plane $z=0$; therefore, it is studied in the half-space $0 \leq z \leq L$. Then, the two-fluid model of plasma is represented by [16, 21, 30, 32, 34, 36];

$\frac{\mathrm{d}\left(n_{\mathrm{i}} v_{z}\right)}{\mathrm{d} z}=v n_{\mathrm{e}}$,

$m_{\mathrm{i}} \frac{\mathrm{d}\left(n_{\mathrm{i}} v_{z}^{2}\right)}{\mathrm{d} z}=e n_{\mathrm{i}} E_{z}-\frac{\mathrm{d} P_{\mathrm{i}}}{\mathrm{d} z}$

$n_{\mathrm{e}}=n_{\mathrm{o}} \exp \left(\frac{e \varphi}{k_{\mathrm{B}} \tau_{\mathrm{e}}}\right)$

$\frac{\mathrm{d}^{2} \varphi}{\mathrm{d} z^{2}}=-\frac{e}{\varepsilon_{\mathrm{o}}}\left(n_{\mathrm{i}}-n_{\mathrm{e}}\right)$.

These equations are the ion continuity and ion momentum conservation equations [Eqs. (1) and (2), respectively], the Boltzmann relation for the electron density [Eq. (3)], and the Poisson's equation [Eq. (4)] in which $n_{\mathrm{i}}$ and $m_{\mathrm{i}}$ are the ion number density and ion mass, respectively, $P_{\mathrm{i}}$ is the static pressure of ions, $E_{z}=-\mathrm{d} \varphi / \mathrm{d} z$ is the $z$-component of electric field, $\varphi$ is the electric potential and $v_{z}$ is the $z$-component of ion velocity. Also, $n_{\mathrm{e}}$ is the electron number density, $n_{\mathrm{o}}=n_{\mathrm{eo}}=n_{\mathrm{io}}$ is the plasma density (ion or electron density) at the plasma center, $k_{\mathrm{B}}$ is the Boltzmann constant, $v$ is the ionization rate and $\varepsilon_{\mathrm{o}}$ stands for the vacuum permittivity.

Besides, the thermal drag of ions is $\mathrm{d} P_{\mathrm{i}} / \mathrm{d} z=\gamma k_{\mathrm{B}} \tau_{\mathrm{i}}\left(n_{\mathrm{i}} / n_{\mathrm{o}}\right)^{(\gamma-1)}\left(\mathrm{d} n_{\mathrm{i}} / \mathrm{d} z\right)$ in which $\gamma$ is the ion polytrophic coefficient. In the case of ion isothermal flow $(\gamma=1)$, the ion thermal drag is simplified to $\mathrm{d} P_{\mathrm{i}} / \mathrm{d} z=k_{\mathrm{B}} \tau_{\mathrm{i}}\left(\mathrm{d} n_{\mathrm{i}} / \mathrm{d} z\right)$ [21, 28, 30, 32].

It is efficient to represent some normalized variables and parameters as; $Z=\left(v / c_{\mathrm{s}}\right) z, T=\tau_{\mathrm{i}} / \tau_{\mathrm{e}}, \alpha=\left(v / c_{\mathrm{s}}\right) \lambda_{\mathrm{D}}$, $\chi=\left(e c_{\mathrm{s}} / k_{\mathrm{B}} \tau_{\mathrm{e}} \nu\right) E_{z}, \eta=-\left(e / k_{\mathrm{B}} \tau_{\mathrm{e}}\right) \varphi, V=v_{z} / c_{\mathrm{s}}, I=n_{\mathrm{i}} / n_{\mathrm{o}}$ and $E=n_{\mathrm{e}} / n_{\mathrm{o}}$ in which $\chi=\mathrm{d} \eta / \mathrm{d} Z$ stands for the normalized electric field, $k_{\mathrm{B}} \tau_{\mathrm{e}}$ is the electron thermal energy, $c_{\mathrm{S}}=\sqrt{k_{\mathrm{B}} \tau_{\mathrm{e}} / m_{\mathrm{i}}}$ introduces the ion sound velocity and $\lambda_{\mathrm{D}}=\sqrt{\varepsilon_{\mathrm{o}} k_{\mathrm{B}} \tau_{\mathrm{e}} / n_{\mathrm{o}} e^{2}}$ defines the Debye length at the plasma center. Therefore, Eqs. (1), (2) and (4) will be turned to;

$\frac{\mathrm{d}(I V)}{\mathrm{d} Z}=E$,

$V \frac{\mathrm{d} V}{\mathrm{~d} Z}=\chi-\frac{E V}{I}-\frac{T}{I} \frac{\mathrm{d} I}{\mathrm{~d} Z}$,

$\alpha^{2} \frac{\mathrm{d}^{2} \eta}{\mathrm{d} Z^{2}}=I-E$,

along with $E=\exp (-\eta)$ for the normalized electron density. These equations must be integrated for proper boundary conditions at the plasma center and on the wall for some different values of the both parameters $T$ and $\alpha$. By integrating from the plasma center $(Z=0)$ to the position $Z=Z_{\mathrm{f}}$ where the floating boundary condition $\eta=\eta_{\mathrm{f}}$ is satisfied, one can simply solve the eigenvalue problem $\left(Z_{\mathrm{f}}\right.$ and $\eta_{\mathrm{f}}$ are called the floating width and floating potential, respectively).

In the special case $\alpha \rightarrow 0$ the Poisson's equation (7) gives rise to $I=E=\exp (-\eta)$ which is named the quasi-neutrality with no space charge. In other words, a neutral plasma and a very thin sheath with positive space charge are formed in front of the electrodes. In order to form a thick sheath layer attached to each electrode in which the Poisson's equation plays a critical role, it is essential to exchange the space coordinate $Z$ to $\zeta=\left(Z-Z_{\mathrm{f}}\right) / \alpha$ with $\zeta$ as the new high resolution coordinate and $Z_{\mathrm{f}}$ as the origin of the new coordinate. In the new coordinate space, set Eqs. (5)-(7) will be exchanged to

$\frac{\mathrm{d}(I V)}{\mathrm{d} \zeta}=\alpha E$,

$V \frac{\mathrm{d} V}{\mathrm{~d} \zeta}=\chi-\alpha \frac{E V}{I}-\frac{T}{I} \frac{\mathrm{d} I}{\mathrm{~d} \zeta}$

$\frac{\mathrm{d}^{2} \varphi}{\mathrm{d} \zeta^{2}}=I-E$,

where $E=\exp (-\varphi)$. There are apparent differences between set Eqs. (5)-(7) and (8)-(10) in the special case $\alpha \rightarrow 0$; however, there is not any difference between them in the case $\alpha>0$ except for the space coordinate. 


\section{Asymptotic and full solutions}

Applying the limit condition $\alpha=0$ in the set Eqs. (5)-(7) or (8)-(10) creates the asymptotic equations. In this case, the plasma asymptotic equations created from Eqs. (5)-(7) are

$\frac{\mathrm{d}\left(I_{\mathrm{o}} V_{\mathrm{o}}\right)}{\mathrm{d} Z}=E_{\mathrm{o}}$

$V_{0} \frac{\mathrm{d} V_{\mathrm{o}}}{\mathrm{d} Z}=\frac{\mathrm{d} \eta_{\mathrm{o}}}{\mathrm{d} Z}-V_{\mathrm{o}}-\frac{T}{I_{\mathrm{o}}} \frac{\mathrm{d} I_{\mathrm{o}}}{\mathrm{d} Z}$,

$I_{\mathrm{o}}=E_{\mathrm{o}}=\exp \left(-\eta_{\mathrm{o}}\right)$

that can be combined and simplified to

$\frac{\mathrm{d} \eta_{\mathrm{o}}}{\mathrm{d} Z}=\frac{2 V_{\mathrm{o}}}{1+T-V_{\mathrm{o}}^{2}}$,

$\frac{\mathrm{d} V_{\mathrm{o}}}{\mathrm{d} Z}=\frac{1+T+V_{\mathrm{o}}^{2}}{1+T-V_{\mathrm{o}}^{2}}$.

Subscription " $o$ ", representing the outer asymptotic solution, has been added to the variables $V, I$ and $\eta$. These equations are just held in the quasi-neutral plasma and their analytical solutions are

$\eta_{\mathrm{o}}=\ln \left(1+\frac{V_{\mathrm{o}}^{2}}{1+T}\right)$

$Z_{\mathrm{o}}=2 \sqrt{1+T} \arctan \left(\frac{V_{\mathrm{o}}}{\sqrt{1+T}}\right)-V_{\mathrm{o}}$,

$I_{\mathrm{o}}=E_{\mathrm{o}}=\exp \left(-\eta_{\mathrm{o}}\right)=\frac{1+T}{1+T+V_{\mathrm{o}}^{2}}$,

These functions are authentic in the interval $0 \leq V_{\mathrm{o}} \leq \sqrt{1+T}$. A singular point, located in $V_{\mathrm{s}}=\sqrt{1+T}=V_{\mathrm{B}}$, is introduced by Eqs. (14) and (15) and is called the general Bohm criterion. The singular point $V_{\mathrm{s}}$ represents the sheath edge where the breakdown of the plasma quasi-neutrality is commenced. Using the general Bohm criterion in Eqs. (16)-(18), the other variables at the sheath edge $\eta_{\mathrm{s}}=\ln 2=0.6931, Z_{\mathrm{s}}=\sqrt{1+T}(\pi / 2-1)$ and $I_{\mathrm{s}}=\exp \left(-\eta_{\mathrm{s}}\right)=1 / 2$ are attained in which the subscription "s" refers to the sheath edge. One can easily find the wellknown amounts $V_{\mathrm{s}}=1$ and $Z_{\mathrm{s}}=\pi / 2-1=0.5708$ in the cold plasmas [18].

On the other hand, applying the condition $\alpha=0$ in the set Eqs. (8)-(10) generates the sheath asymptotic equations
$\frac{\mathrm{d}\left(I_{\mathrm{i}} V_{\mathrm{i}}\right)}{\mathrm{d} \zeta}=0$,

$V_{\mathrm{i}} \frac{\mathrm{d} V_{\mathrm{i}}}{\mathrm{d} \zeta}=\chi_{\mathrm{i}}-\frac{T}{I_{\mathrm{i}}} \frac{\mathrm{d} I_{\mathrm{i}}}{\mathrm{d} \zeta}$

$\frac{\mathrm{d} \chi_{\mathrm{i}}}{\mathrm{d} \zeta}=I_{\mathrm{i}}-E_{\mathrm{i}}$

$\frac{\mathrm{d} \eta_{\mathrm{i}}}{\mathrm{d} \zeta}=\chi_{\mathrm{i}}$

The additional subscription " $i$ " has been added to the variables $V, I, \eta$ and $\chi$ to introduce the inner asymptotic solution. These equations are just held in the positive spacecharge sheath. These equations are not included the ionization term any more. Using Eqs. (19) and (22) in Eq. (20) and integrating of the obtained equation from the sheath edge to a general point in the sheath region results to;

$\left(\frac{V_{\mathrm{i}}}{V_{\mathrm{s}}}\right)^{2}-\frac{2 T}{V_{\mathrm{s}}^{2}} \ln \left(\frac{V_{\mathrm{i}}}{V_{\mathrm{s}}}\right)=2 \frac{\eta_{\mathrm{i}}-\eta_{\mathrm{s}}}{V_{\mathrm{s}}^{2}}+1$,

$\left(\frac{I_{\mathrm{s}}}{I_{\mathrm{i}}}\right)^{2}-\frac{2 T}{V_{\mathrm{s}}^{2}} \ln \left(\frac{I_{\mathrm{s}}}{I_{\mathrm{i}}}\right)=2 \frac{\eta_{\mathrm{i}}-\eta_{\mathrm{s}}}{V_{\mathrm{s}}^{2}}+1$,

for the ion velocity and ion density as functions of the electric potential $\eta_{\mathrm{i}}$. These equations are lessened to the specified relations;

$V_{\mathrm{i}}=V_{\mathrm{s}}\left(2 \frac{\eta_{\mathrm{i}}-\eta_{\mathrm{s}}}{V_{\mathrm{s}}^{2}}+1\right)^{1 / 2}$,

$I_{\mathrm{i}}=I_{\mathrm{s}}\left(2 \frac{\eta_{\mathrm{i}}-\eta_{\mathrm{s}}}{V_{\mathrm{s}}^{2}}+1\right)^{-1 / 2}$,

in a nonthermal plasma [16].

Following some separate discussions on the both plasma asymptotic limit (introduced by the quasi-neutrality condition) and sheath asymptotic limit (with no ionization), it is the time to study the behavior of the whole plasma in the body and near the wall simultaneously. It means that the plasma Eqs. (5)-(7) should be solved for some small finite amounts of $\alpha$. It is easy to verify that set Eqs. (5)-(7) give rise to the first-order differential equations

$\frac{\mathrm{d} V}{\mathrm{~d} Z}=\frac{1}{V^{2}-T}\left(V \chi-\frac{E}{I} V^{2}-T \frac{E}{I}\right)$, 
$\frac{\mathrm{d} I}{\mathrm{~d} Z}=\frac{2 V E-I \chi}{V^{2}-T}$

$\frac{\mathrm{d} \chi}{\mathrm{d} Z}=\frac{I-E}{\alpha^{2}}$,

$\frac{\mathrm{d} \eta}{\mathrm{d} Z}=\chi$

Numerical solution of these equations, from the plasma center to the wall, is the main goal of the rest of this paper. The required boundary conditions at the plasma center are computed using power series expansions for the variables $I, V$ and $\eta$ at that point. Evidently, the plasma variables $I$ and $\eta$ are even functions of the space coordinate $Z$, whereas ion velocity $V$ is an odd function. Therefore, they might be introduced by

$I \approx i_{1}+i_{2} Z^{2}$

$\eta \approx\left(f_{1}+f_{2} Z^{2}\right) Z^{2}$

$V \approx\left(v_{1}+v_{2} Z^{2}\right) Z$

and consequently

$\chi=\frac{\mathrm{d} \eta}{\mathrm{d} Z} \approx\left(2 f_{1}+4 f_{2} Z^{2}\right) Z$,

$E=\exp (-\eta) \approx 1-f_{1} Z^{2}+\left(\frac{f_{1}^{2}}{2}-f_{2}\right) Z^{4}$,

in which there are six unknown coefficients $i_{1}, i_{2}, f_{1}, f_{2}, v_{1}$ and $v_{2}$ that must be determined. Applying these approximate relations in Eqs. (27)-(30) and comparing the coefficients of the two lowest order in $Z$ in the both sides of the acquired relations result in,

$i_{1}=1+\frac{2\left(i_{1}^{3}+12 \alpha^{2}\right) \alpha^{2}}{\left[4 i_{1}^{3}+(T-3) i_{1}^{2}+2(4 T+3) \alpha^{2}\right] i_{1}^{2}}$,

and

$i_{2}=\frac{f_{1} i_{1}-v_{1}}{T}, \quad v_{1}=\frac{1}{i_{1}}, \quad f_{1}=\frac{i_{1}-1}{2 \alpha^{2}}$,

$f_{2}=\frac{i_{2}+f_{1}}{12 \alpha^{2}}, \quad v_{2}=-\frac{f_{1}+3 i_{2} v_{1}}{3 i_{1}}$.

Starting with $i_{1}=1$ as the initial amount and utilizing relation (31) iteratively, one can find the principle factor $i_{1}$; the other coefficients are determined using $i_{1}$.

By finding the boundary conditions in a near vicinity of the plasma center, say $Z=0.01$, one can proceed to solve
Eqs. (27)-(30). But, it is apparent that there is a singularity in these equations. It is essential to transmit the singularity point smoothly when solving the equations. To cross this singular point correctly and smoothly, an approximate technique are utilized. In this method, a velocity interval is symmetrically chosen around the singular point $V=\sqrt{T}$ in which the both following equations are worked out instead of Eqs. (27) and (28)

$\frac{\mathrm{d} V}{\mathrm{~d} Z}=\frac{\chi}{V}-\frac{E}{I}-\frac{T}{V I} \frac{\mathrm{d} I}{\mathrm{~d} Z}$,

$\frac{\mathrm{d} I}{\mathrm{~d} Z}=\frac{E}{V}-\frac{I}{V} \frac{\mathrm{d} V}{\mathrm{~d} Z}$

Equations (32) and (33) are the direct consequences of Eqs. (5) and (6). In this method, the gradient of the ion density $I$ and the ion velocity $V$ have been specified just before the interval; therefore, they can be used in Eqs. (32) and (33) to find $I$ and $V$ and their gradients in the next step and this sequences stay on to the end of the interval. Again, we will come back to the singular Eqs. (27) and (28) at the end of the interval. It is important to mention that there is no need to replace Eqs. (29) and (30) since they are not singular.

In order to find the full solution of Eqs. (27)-(30), it is essential to end the computations at a suitable location. Floating point, in which the ion directional current is equal to the electron random current, is selected as the right location. The floating point is specified by; $n_{\mathrm{if}} v_{z \mathrm{f}}=n_{\mathrm{ef}} c_{\mathrm{e}} / 4$ in which $n_{\text {if }}$ is the ion density, $v_{z \mathrm{f}}$ is the ion velocity and $n_{\mathrm{ef}}$ is the electron density at that point, and $c_{\mathrm{e}}=\sqrt{8 k_{\mathrm{B}} T / \pi m_{\mathrm{e}}}$ is the electron thermal velocity [16]. The recent floating relation can be rewrite in the normalized form as follows

$I_{\mathrm{f}} V_{\mathrm{f}}=E_{\mathrm{f}} \sqrt{\frac{m_{\mathrm{i}}}{2 \pi m_{\mathrm{e}}}}=\sqrt{\frac{m_{\mathrm{i}}}{2 \pi m_{\mathrm{e}}}} \exp \left(-\eta_{\mathrm{f}}\right)$,

leading to

$\eta_{\mathrm{f}}=\ln \left(\frac{\beta}{I_{\mathrm{f}} V_{\mathrm{f}}}\right)$,

in which, $\beta=\sqrt{m_{\mathrm{i}} / 2 \pi m_{\mathrm{e}}}$. We have done the computations with $\beta=108.0832$ calculated for the electropositive gas Argon. Relation (34) is known as the floating condition and $\eta_{\mathrm{f}}$ is called the floating potential.

As noted at the outset, in the plasma asymptotic limit (with $\alpha=0$ ), the sheath region is infinitely thin. Then, in the full solution approach and for $\alpha=0$, all of the variables behave such as the step function. Therefore, in this limiting case, using the sheath width $Z_{\mathrm{s}}=\sqrt{1+T}(\pi / 2-1)$ as the floating width $Z_{\mathrm{f}}$ is reasonable [16]. Moreover, relation (24) results in 
$I_{\mathrm{f}}=I_{\mathrm{s}}\left[1+2 \frac{\eta_{\mathrm{f}}-\eta_{\mathrm{s}}}{V_{\mathrm{s}}^{2}}+\frac{2 T}{V_{\mathrm{s}}^{2}} \ln \left(\frac{I_{\mathrm{s}}}{I_{\mathrm{f}}}\right)\right]^{-1 / 2}$.

By using relation (35), one can determine the floating ion density $I_{\mathrm{f}}$ by iteration and starting with $I_{\mathrm{f}}=I_{\mathrm{s}}\left[1+2\left(\eta_{\mathrm{f}}-\eta_{\mathrm{s}}\right) / V_{\mathrm{s}}^{2}\right]^{-1 / 2}$ as the initial value. Finally, using the relation $V_{\mathrm{f}} I_{\mathrm{f}}=V_{\mathrm{s}} I_{\mathrm{s}}$ it is easy to find the floating ion velocity $V_{\mathrm{f}}$.

The spatial variations of the plasma variables in the full solution approach are shown in Fig. 1. It is clear from this figure that the spatial distribution of the ion density $I$ is broken at the beginning of the ion velocity interval selected symmetrically around the singular point $V=\sqrt{T}$; similarly, the spatial distribution of the ion velocity $V$ suffers a small rippling at that point. At the end point of the interval, however, the both distributions display complete smoothness. Also there is not any unevenness or breaking at the spatial distributions of the both electric potential $\eta$ and electron density $E$.

Figures 2, 3, 4, 5, 6, 7 and 8 exhibit some of the plasma variables including; the floating ion density $I_{\mathrm{f}}$, floating electron density $E_{\mathrm{f}}$, central ion density $I_{c}$, floating electric potential $\eta_{\mathrm{f}}$, spatial distribution of electric potential $\eta$, floating ion velocity $V_{\mathrm{f}}$ and floating width $X_{\mathrm{f}}$ (extended from the plasma center to the floating point) as functions of the ion temperature $T$ and smallness parameter $\alpha$, respectively. The equations have been solved for $\alpha=0$ to 0.2 and $T=0$ to 1 ..

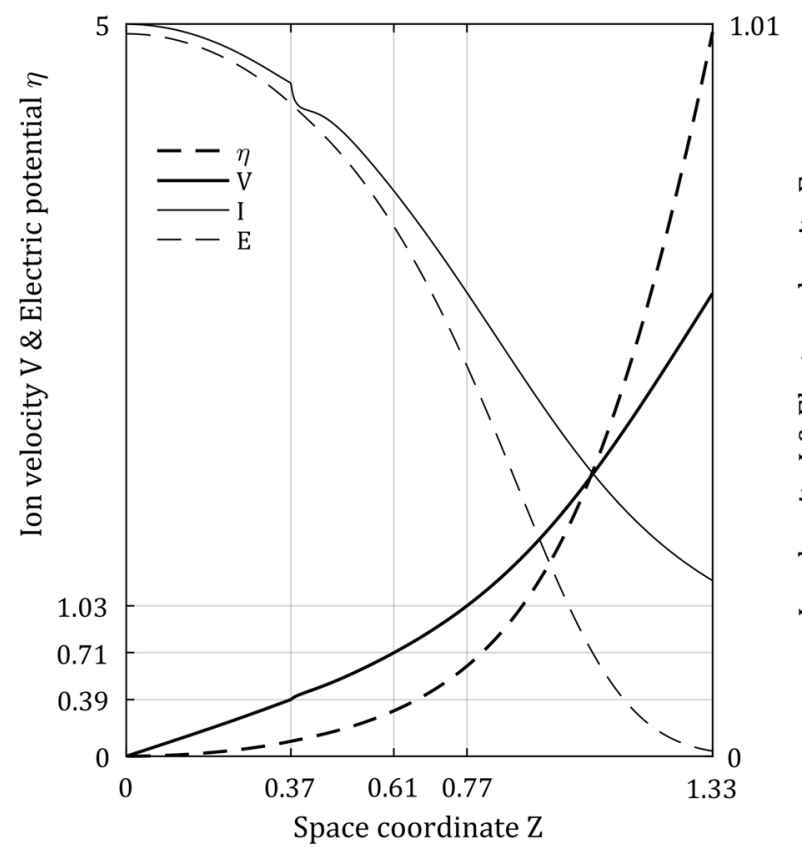

Fig. 1 The spatial distributions of ion density $I$, electron density $E$, ion velocity $V$ and electric potential $\eta$ as functions of the space coordinate $Z$ for $T=0.5$ and $\alpha=0.1$. The quantities on the $V$ axis are the singular point $V=\sqrt{T} \approx 0.71$ and the symmetrical interval around it (and their corresponding locations on the $Z$-axis)

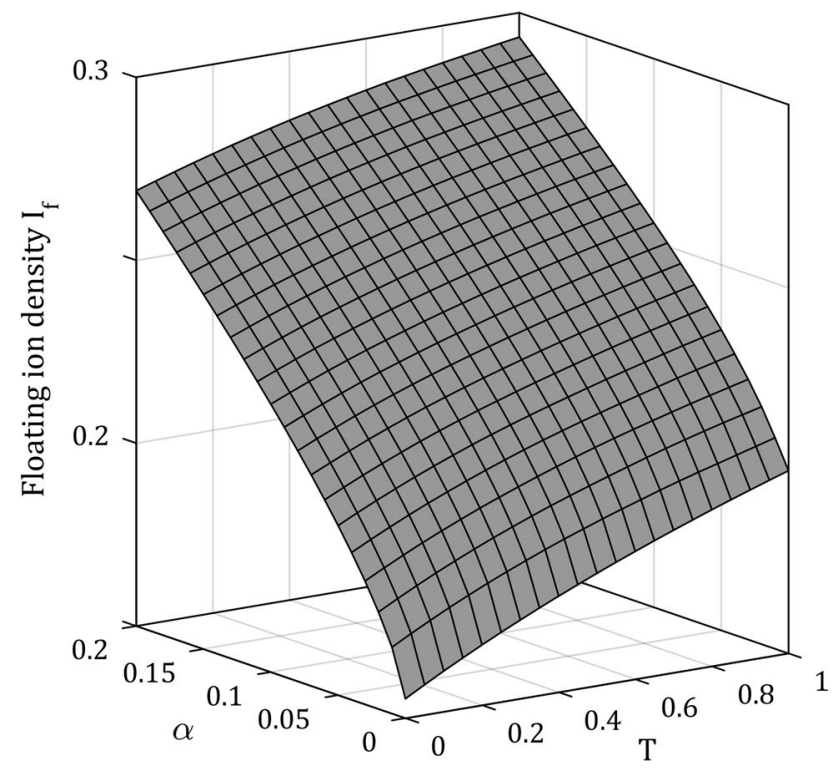

Fig. 2 Floating ion density $I_{\mathrm{f}}$ as a function of the ion temperature $T$ and smallness parameter $\alpha$

According to Figs. 2 and 3, floating ion and electron densities are increasing functions of the both ion temperature and smallness parameter. Since smallness parameter $\alpha$ is the only factor for plasma generation in the boundary layer, so it is reasonable that the plasma density become an increasing function of this parameter throughout the boundary layer [21].

On the other hand, Fig. 4 shows that the ion density at the plasma center decreases by rising the ion temperature, while

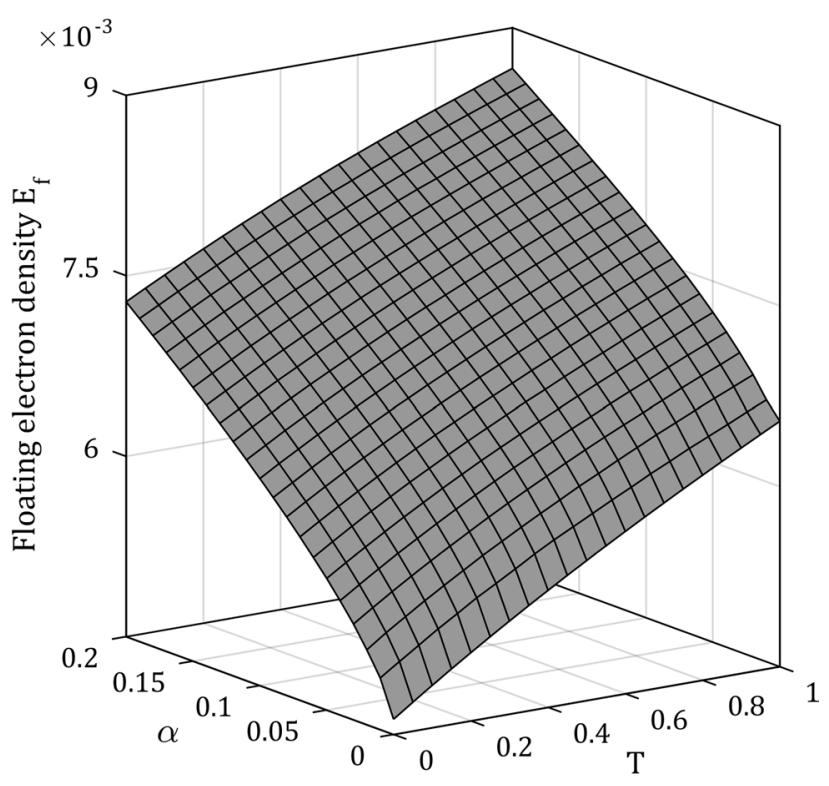

Fig. 3 Floating electron density $E_{\mathrm{f}}$ as a function of the ion temperature $T$ and smallness parameter $\alpha$ 


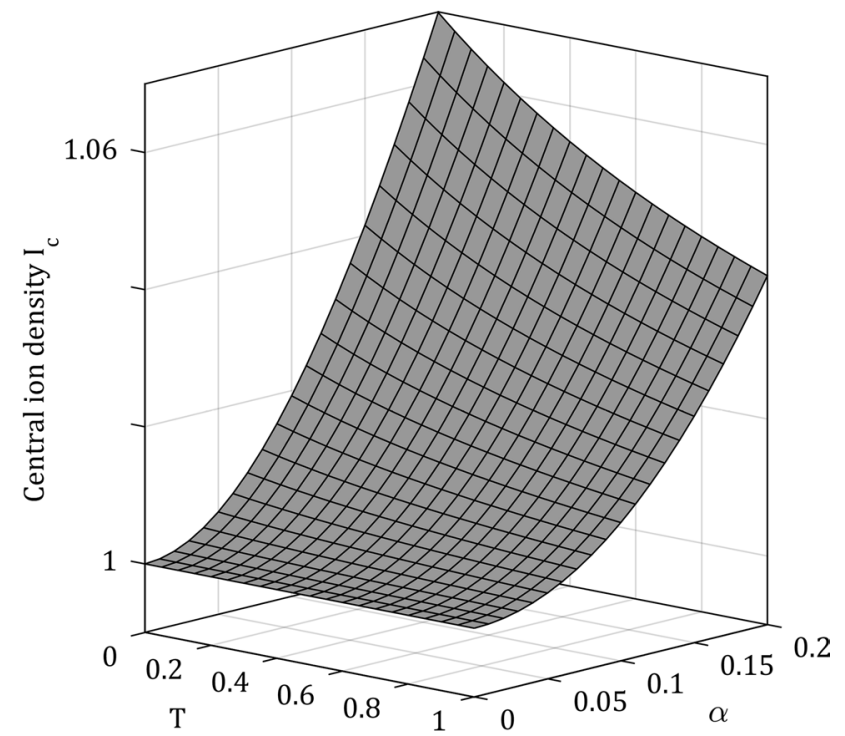

Fig. 4 The ion density at the plasma center $I_{c}$ as a function of the ion temperature $T$ and smallness parameter $\alpha$

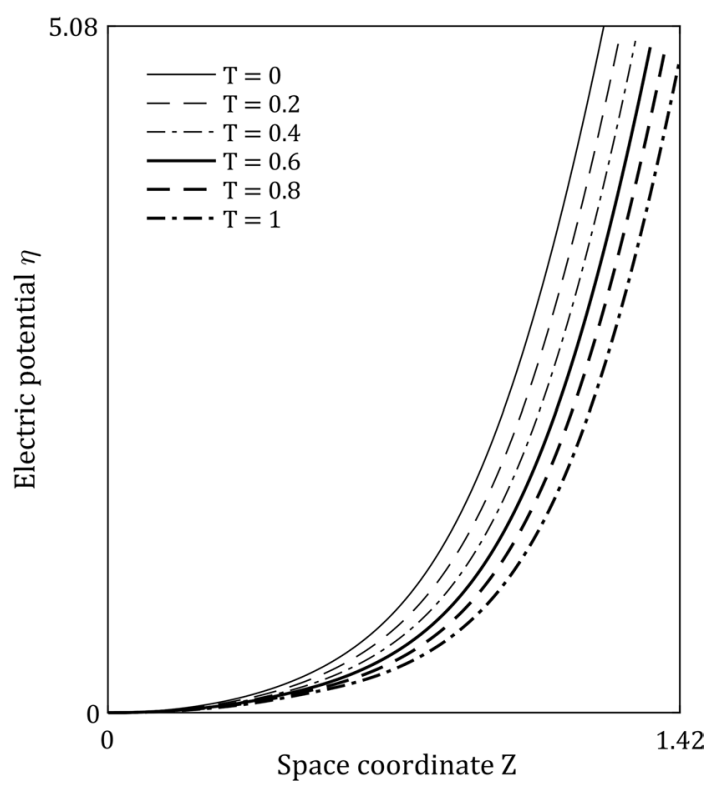

Fig. 5 Spatial distribution of electric potential $\eta$ for $\alpha=0.1$ and some different values of ion temperature $T$

Fig. 2 displays that the ion temperature raises the floating ion density. It means that the ion temperature expands the plasma and extends it from the center to the outside of the plasma. Moreover, these figures show that smallness parameter $\alpha$ intensifies the ion temperature effect [21].

Figure 5 exhibits the spatial distribution of electric potential $\eta$ from the plasma center to the floating point for $\alpha=0.1$ and some values of ion temperature. It is apparent that the ion temperature decreases the electric potential distribution throughout the boundary layer. Also Fig. 6 shows that the

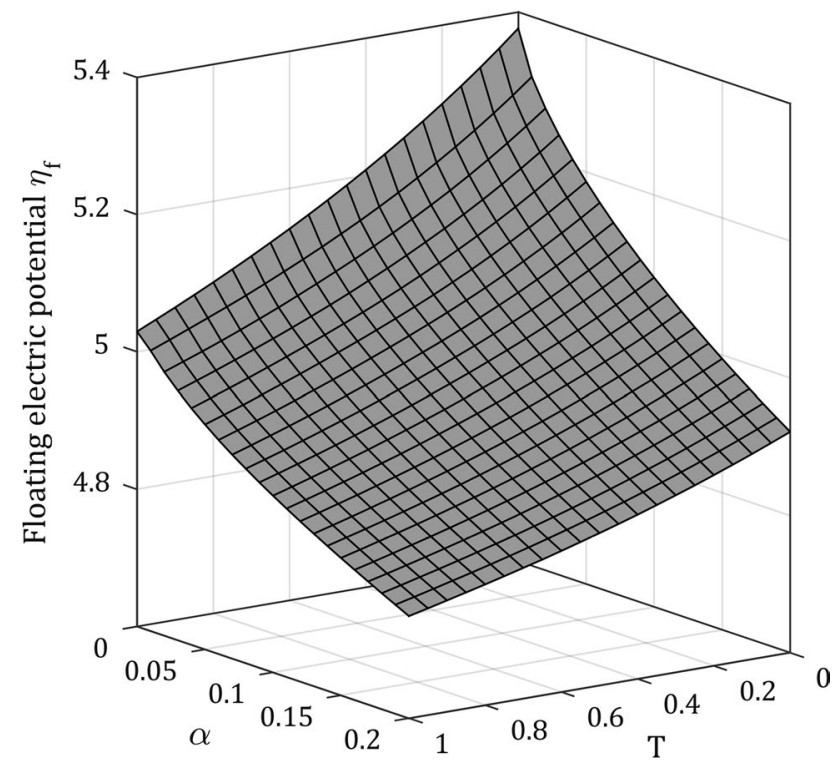

Fig. 6 Floating electric potential $\eta_{\mathrm{f}}$ as a function of the ion temperature $T$ and smallness parameter $\alpha$

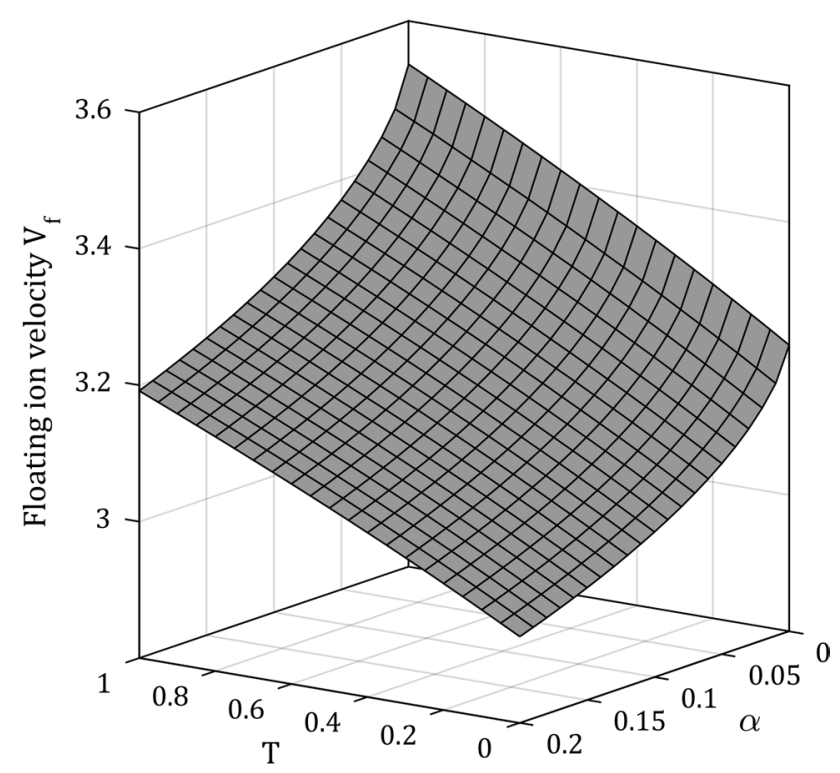

Fig. 7 Floating ion velocity $V_{\mathrm{f}}$ as a function of the ion temperature $T$ and smallness parameter $\alpha$

floating electric potential $\eta_{\mathrm{f}}$ is a descending function of the both ion temperature and smallness parameter. It means that the more plasma heating and the more plasma generating, the less floating potential.

Figure 7 displays the dependency of the floating ion velocity $V_{\mathrm{f}}$ to the ion temperature $T$ and smallness parameter $\alpha$. According to this figure, ion temperature increases and smallness parameter decreases the ion velocity and ion kinetic energy on the floating wall. Indeed, growing the ion 


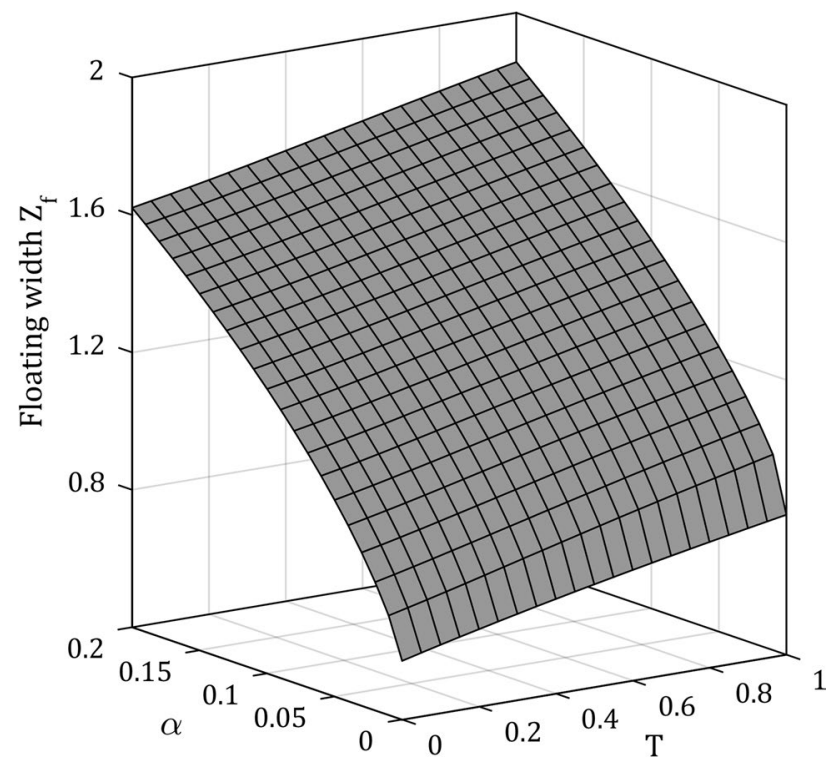

Fig. 8 Floating width $Z_{\mathrm{f}}$ as a function of the ion temperature $T$ and smallness parameter $\alpha$

temperature amplifies the thermal force on the ions and accelerates them outward to the wall. As a result of this process, the ion density on the floating wall increases and causes to pull out more electrons from the plasma center to the wall according to the electrostatic absorption. Moreover, the increased electron density on the floating wall reduces the floating potential according to the Boltzmann relation.

Finally, it can be seen from Fig. 8 that the ion temperature $T$ and smallness parameter $\alpha$ increase the floating width $Z_{\mathrm{f}}$. This is a result of growing the ion and electron density by increasing the ion temperature and the ion generation rate (or smallness parameter $\alpha$ ) in the boundary layer [21].

It is important to note that the width of the ion velocity interval adjusted symmetrically around the singular point $\sqrt{T}$ has no effect on the floating variables and the general aspects of the boundary layer structure. Indeed, the velocity interval has been specified at the least possible amount to ensure the least unevenness in the spatial distribution of variables.

\section{Summary and results}

A fluidal treatment has been used to investigate how the ion temperature and the ion production rate affect on the plasma boundary layer structure as well as on the floating variables. First, the thermal plasma equations have been stated in two plasma and sheath scales and in the special case $\alpha=0$ (no ion generation in the boundary layer) have been analytically solved. Furthermore, in the more common case $\alpha \gtrsim 0$ the equations have been numerically solved from the plasma center to the wall in the plasma scale which is named the full solution approach.

In the full solution approach, the warm plasma equations including the smallness parameter $\alpha$ and ion temperature $T$ which are singular at $V=\sqrt{T}$ have been analyzed in the plasma scale. In order to pass the singular point, an approximate method has been introduced. On the basis of this procedure, the full solution of the plasma equations introduces a breaking point near the singular point in the ion density distribution. This fracture is appeared much more faintly in the ion velocity distribution and is disappeared in the electric potential distribution.

The computations show that the ion temperature increases the floating width and all of the other variables at the floating point except the electric potential which is decreased by temperature. In addition, floating ion and electron densities and floating width are in direct relation with the ion generation rate (or smallness parameter) but the ion velocity and electric potential at the floating point are descending functions of this parameter.

Acknowledgements This study was supported by Islamic Azad University, Borujerd Branch, Iran. The authors would like to acknowledge staffs of the university.

Open Access This article is licensed under a Creative Commons Attribution 4.0 International License, which permits use, sharing, adaptation, distribution and reproduction in any medium or format, as long as you give appropriate credit to the original author(s) and the source, provide a link to the Creative Commons licence, and indicate if changes were made. The images or other third party material in this article are included in the article's Creative Commons licence, unless indicated otherwise in a credit line to the material. If material is not included in the article's Creative Commons licence and your intended use is not permitted by statutory regulation or exceeds the permitted use, you will need to obtain permission directly from the copyright holder. To view a copy of this licence, visit http://creativecommons.org/licenses/by/4.0/.

\section{References}

1. Lieberman, M.A., Lichtenberg, A.J.: Principles of Plasma Discharges and Materials Processing, 1st edn. Wiley, New York (1994)

2. Hutchinson, I.H.: Principles of Plasma Diagnostics, 2nd edn. Cambridge University Press, Cambridge (2002)

3. Qin, S., Ghan, C.: Plasma immersion ion implantation doping experiments for microelectronics. Vac. Sci. Technol. B 12, 962 (1994)

4. Stangeby, P.C.: Plasma Boundary of Magnetic Fusion Devices, 1st edn. Institute of Physics Publishing, Bristol (2000)

5. Pico, C.A., Lieberman, M.A., Cheung, N.W.: Pmos integrated circuit fabrication using $\mathrm{BF}_{3}$ plasma immersion ion implantation. Electron. Mater. 21, 75 (1992)

6. Conrad, J.R., Radtke, J., Dodd, R.A., Worzala, F.J.: Plasma source ion-implantation technique for surface modification of materials. Appl. Phys. 62, 4591 (1987) 
7. Qin, S., McGruer, N., Chan, C., Warner, K.: Plasma immersion ion implantation doping using a microwave multipolar bucket plasma. IEEE Trans. Electron Devices 39, 2354 (1992)

8. Kaganovich, I.D.: How to patch active plasma and collisionless sheath: a practical guide. Phys. Plasmas 9, 4788 (2002)

9. Godyak, A.V., Sternberg, N.: Good news: you can patch active plasma and collisionless sheath. IEEE Trans. Plasma Sci. 31, 303 (2003)

10. Franklin, R.N.: Comment on "on the consistency of the collisionless sheath model" [phys. plasmas 9, 4427 (2002)]. Phys. Plasmas 10, 4589 (2003)

11. Riemann, K.-U.: "Comments on" on asymptotic matching and the sheath edge. IEEE Trans. Plasma Sci. 32, 2265 (2004)

12. Godyak, A.V., Sternberg, N.: On the consistency of the collisionless sheath model. Phys. Plasmas 9, 4427 (2002)

13. Riemann, K.-U.: Comment on "on the consistency of the collisionless sheath model" [phys. plasmas 9, 4427 (2002)]. Phys. Plasmas 10, 3432 (2003)

14. Benilov, M.S.: Method of matched asymptotic expansions versus intuitive approaches: calculation of space-charge sheaths. IEEE Trans. Plasma Sci. 31, 678 (2003)

15. Sternberg, N., Godyak, A.V.: On asymptotic matching and the sheath edge. IEEE Trans. Plasma Sci. 31, 665 (2003)

16. Franklin, R.N., Snell, J.: The low-pressure positive column in electronegative gases including space charge-matching plasma and sheath. Phys. D Appl. Phys. 31, 2532 (1998)

17. Sternberg, N., Godyak, A.V.: Reply to comments on "on asymptotic matching and the sheath edge". IEEE Trans. Plasma Sci. 32, 2271 (2004)

18. Riemann, K.-U., Seebacher, J., Sr, D.D.T., Kuhn, S.: The plasmasheath matching problem. Plasma Phys. Controll. Fusion 47, 1949 (2005)

19. Franklin, R.N.: Where is the sheath edge? Phys. D Appl. Phys. 37, 1342 (2004)

20. Franklin, R.N.: Plasma Phenomena in Gas Discharges, 1st edn. Clarendon, Oxford (1976)

21. Valentini, H.-B.: Removal of singularities in the hydrodynamic description of plasmas including space-charge effects, several species of ions and non-vanishing ion temperature. J. Phys. D Appl. Phys. 21, 311-21 (1988)

22. Das, G.C., Singha, B., Chutia, J.: Characteristic behavior of the sheath formation in thermal plasma. Phys. Plasmas 6, 3685 (1999)

23. Palop, J.I.F., Ballesteros, J., Hernandez, M.A., Crespo, R.M., del Pino, S.B.: Influence of the positive ion thermal motion on the stratified presheath in electronegative plasmas. J. Phys. D Appl. Phys. 37, 863 (2004)

24. Crespo, R.M., Palop, J.I.F., Hernandez, M.A., Ballesteros, J.: Analytical fit of the I-V probe characteristic for finite ion temperature values: justification of the radial model applicability. J. Appl. Phys. 95, 2982 (2004)
25. Ballesteros, J., Palop, J.I.F., Hernandez, M.A., Crespo, R.M.: Influence of the positive ion temperature in cold plasma diagnosis. Appl. Phys. Lett. 89(10), 101501 (2006)

26. Palop, J.I.F., Ballesteros, J., Crespo, R.M., Hernandez, M.A.: Sheath analysis in collisional electronegative plasmas with finite temperature of positive ions. J. Phys. D Appl. Phys. 41, 235201 (2008)

27. Alterkop, B., Goldsmith, S., Boxman, R.L.: Presheath in fully ionized collisional plasma in a magnetic field. Contrib. Plasma Phys. 45, 485 (2005)

28. Ghomi, H., Khoramabadi, M., Shukla, P.K., Ghorannevis, M.: Plasma sheath criterion in thermal electronegative plasmas. Appl. Phys. 108, 063302 (2010)

29. Khoramabadi, M., Ghomi, H., Shukla, P.K.: Numerical investigation of the ion temperature effects on magnetized dc plasma sheath. Appl. Phys. 109, 073307 (2011)

30. Hatami, M.M., Shokri, B.: Bohm's criterion in a collisional magnetized plasma with thermal ions. Phys. Plasmas 19, 083510 (2012)

31. Khoramabadi, M., Ghomi, H., Shukla, P.K.: The Bohm-sheath criterion in plasmas containing electrons and multiply charged ions. J. Plasma Phys. 79, 267 (2013)

32. Aslaninejad, M., Yasserian, K.: Singularity and Bohm criterion in hot positive ion species in the electronegative ion sources. Phys. Plasmas 23, 053505 (2016)

33. Khoram, M., Ghomi, H., NavabSafa, N.: Ion temperature and gas pressure effects on the magnetized sheath dynamics during plasma immersion ion implantation. Phys. Plasmas 23, 033511 (2016)

34. Gyergyek, T., Kovacic, J.: Numerical analysis of ion temperature effects to the plasma wall transition using a one-dimensional two-fluid model. I. Finite debye to ionization length ratio. Phys. Plasmas 24, 063505 (2017)

35. Khoram, M.: The plasma and sheath asymptotic solutions along with the full solution of the plasma equations including the ion isothermal flow. IEEE Trans. Plasma Sci. 47, 1704-12 (2019)

36. Friedman, H.W., Levi, E.: Singularities of the two-fluid plasma equations and their relation to boundary conditions. Phys. Fluids 10, 1499 (1967)

37. Ingold, J.H.: Two-fluid theory of the positive column of a gas discharge. Phys. Fluids 15, 75 (1972)

38. Valentini, H.B., Kaiser, D.: The singularity of the two-fluid plasma equations, its relations to boundary conditions, and the numerical solution of these equations. Phys. Plasmas 24, 123508 (2017)

Publisher's Note Springer Nature remains neutral with regard to jurisdictional claims in published maps and institutional affiliations. 